\section{Giant leiomyoma of the urinary bladder: A case report}

\author{
Khalid Atallah, ${ }^{1}$ Omar Hamdy, ${ }^{1}$ \\ Gehad Ahmed Saleh, ${ }^{2}$ \\ Farida A Shokeir, ${ }^{3}$ Yasmin Laimon, ${ }^{3}$ \\ Ahmed Hassan, ${ }^{1}$ Khaled Abdel Wahab ${ }^{1}$ \\ ${ }^{1}$ Surgical Oncology Unit, Oncology \\ Center; ${ }^{2}$ Radiology Department; \\ ${ }^{3}$ Pathology Department, Faculty of \\ Medicine, Mansoura University, \\ Mansoura, Egypt
}

\begin{abstract}
Urinary bladder leiomyoma is a rare tumour accounting for less than $0.5 \%$ of all urinary bladder tumours. Till now, less than 250 cases were documented with variable sizes, most of them were less than $10 \mathrm{~cm}$ in maximum diameter. Here we present a 68 year-old female patient with urinary bladder giant leiomyoma measuring about $13 \mathrm{~cm}$. She presented with right loin pain. Postcontrast computed tomography of the abdomen and pelvis revealed a large posterolateral right-sided urinary bladder mass with moderate right hydroureteronephrosis. It was managed by partial cystectomy. The patient had an uneventful postoperative course. Postoperative pathological examination of the specimen confirmed giant leiomyoma of the urinary bladder.
\end{abstract}

\section{Introduction}

Benign urinary bladder mesenchymal tumours account for $1-5 \%$ of all bladder tumours. Leiomyoma is the most common type. Yet, it is a rare tumour that accounts only for less than $0.5 \%$ of all bladder tumours. ${ }^{1}$ Other benign mesenchymal tumours include myoma, rhabdomyoma, fibroma, angioma, osteoma and myxoma. ${ }^{2}$

Till now, the exact etiology of leiomyomas is unknown, but many theories have been suggested. Some authors suggest that estrogen has a role in the growth of leiomyomas due to the presence of identifiable estrogen receptors in leiomyomatous tissues. ${ }^{3}$ The most common presenting symptoms are obstructive symptoms (49\%), followed by irritating symptoms $(38 \%)$. Flank pain $(13 \%)$ and hematuria $(11 \%)$ are less frequent presentations. Asymptomatic silent presentation is occasional as well $(20 \%))^{4}$

Management of these patients included either conservative management, transurethral resection, enucleation, partial cys- tectomy or even cystectomy in some patients. ${ }^{5}$ Although the clinical treatments are different for this type of disease, the prognosis is generally excellent. ${ }^{6}$

Several reviews were published with

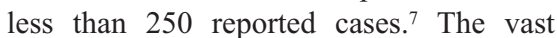
majority of the reported cases had masses less than $10 \mathrm{~cm}$ in diameter. ${ }^{5}$ The largest reported bladder leiomyoma was described in 1998 and measured $23 \mathrm{~cm}$ in its greatest dimension. ${ }^{8}$ Here we report a rare presentation with a giant leiomyoma of the urinary bladder that measured $13 \mathrm{~cm}$ in its greatest dimension.

\section{Case Report}

A sixty-eight-year-old hypertensive lady presented with right loin pain for few weeks. There were neither hematuria nor irritative lower urinary tract symptoms. The patient had a surgical history of right renal stone extraction a few years before.

Pelvi-abdominal ultrasonography (US) revealed moderate back pressure changes on the right kidney and ureter down to large pelvic solid mass related to the posterior wall of the urinary bladder suggesting malignancy. The patient then was referred to the surgical oncology unit in the Oncology center, Mansoura University. Post-contrast abdominal and pelvic Computed Tomography (CT) was requested. It revealed a large heterogeneously enhancing soft tissue mass at the right side of the pelvis involving the right postero-lateral wall of the urinary bladder as well as the related ureteric orifice with subsequent moderate right hydroureteronephrosis. It measured 13x10x11 cm AP x W x H respectively. It was seen compressing and displacing the uterine body and cervix to the left side with a clear fat plane in-between and contacting the right external iliac vessels without invasion. In the delayed phase, no contrast leakage was detected denoting intact UB wall (Figure 1).

Examination under anesthesia revealed a large mobile pelvic mass. Rigid cystoscopy revealed evidence of a large mass at the right lateral bladder wall obstructing the right ureteric orifice. Trial of ureteric stenting failed due to marked compression on the ureteric orifice by the tumour.

Multiple punch biopsies were taken revealing benign proliferative lesion with squamous metaplasia. Then US-guided core needle biopsy was performed. Histopathological examination of the biopsy revealed smooth muscle proliferation suggestive of leiomyoma. After the multidisciplinary team meeting, the decision was to surgically excise the tumour after patient
Correspondence: Khalid Atallah, Surgical Oncology Unit, Oncology Center, Mansoura University, Gehan Street, 35511 Mansoura, Egypt.

Tel.: +201003551338

E-mail: Khalid_atallah@mans.edu.eg

Key words: Bladder; leiomyoma; benign tumours; giant.

Contributions: KA was responsible for data collection, writing, and revision. GAS was responsible for the radiology part. FAS and YL were responsible for data collection and writing the pathology part. $\mathrm{OH}$ was responsible for step-by-step revision. KAW was responsible for the operative part and supervision. All authors have read and approved the final manuscript.

Conflict of interest: The authros have no conflict of interest to declare.

Availability of data and materials: All data generated or analyzed during this study are included in this published article.

Ethics approval and consent to participate: All procedures performed were following the ethical standards of the institutional research committee and with the 1964 Helsinki Declaration and its later amendments. Specific ethical committee approval is not required in case reports. Verbal informed consent was obtained from the patient for publication.

Received for publication: 22 April 2021.

Revision received: 17 September 2021.

Accepted for publication: 22 September 2021.

This work is licensed under a Creative Commons Attribution NonCommercial 4.0 License (CC BY-NC 4.0).

(C) Copyright: the Author(s), 2021

Licensee PAGEPress, Italy

Urogynaecologia 2021; 33:270

doi:10.4081/uij.2021.270

preparation. Exploration was done through a midline incision. A large mobile mass was found within the right side of the posterior wall of the urinary bladder. The right ureter was compressed by the tumour with subsequent proximal dilatation. Partial cystectomy was performed. The defect of the bladder wall was repaired in layers. The patient was discharged on the third postoperative day with the urinary catheter. A urinary catheter was kept for 2 weeks. The postoperative course was uneventful except for small operative bed collection that was managed conservatively.

After surgery, the presenting symptoms declined and the right hydroureteronephro- 
sis was relieved. No recurrence occurred till the time of publication.

Postoperative histopathological examination of the specimen revealed benign tumoral proliferation of bland-looking smooth muscle fibers with cigar-shaped nuclei, arranged in a whorly pattern. No detected mitosis, necrosis, atypia, or malignancy in the dissected specimen. The pathological diagnosis was urinary bladder leiomyoma (Figure 2).

\section{Discussion}

Leiomyoma is the most common benign mesenchymal urinary bladder tumour. It accounts for less than $0.5 \%$ of all bladder tumours. The largest review discussing bladder leiomyoma included 90 cases. Cases had female predilection $(75.6 \%)$ and a mean age of 45.3 years. ${ }^{9}$

Cornella's review included 23 cases of bladder leiomyoma collected in the period between 1940 and 1995..$^{10}$ Another review included 21 cases collected from 2012 to 2017. (6) Only one patient in each review had a tumour size of more than $10 \mathrm{~cm}$.

They may be endovesical, intramural, and extravesical. The endovesical location is the most common one and it corresponds to $63-86 \%$ of cases, while intramural leiomyomas are present in 3-7\% and extravesical in $11-30 \% .^{11}$

Generally, larger leiomyomas have more symptoms. However, bladder leiomyoma smaller than $1.4 \mathrm{~cm}$ in diameter can cause pain and urinary retention. ${ }^{12}$ This phenomenon is mainly attributed to the location of the tumour. A leiomyoma that is located in the bladder neck may cause more severe symptoms compared with a leiomyoma that is located in the bladder wall. ${ }^{6}$

Various radiologic methods are used to diagnose this disease entity including intravenous pyelography, ultrasonography, CT, CT Urography and MRI. ${ }^{13}$ Both CT and MRI offer excellent delineation of the tumor, However, MRI is the preferred method of choice because it offers more superior soft-tissue contrast \& resolution with more precise detection of the site of origin. ${ }^{14}$

Pathologically, Urinary bladder leiomyoma resembles that of the uterus. On histopathologic examination, it shows spindle cells proliferation with eosinophilic cytoplasm and centrally located cigar-shaped nuclei within a fibrous stroma. ${ }^{15}$ While, leiomyosarcoma, on the other hand, may also show few mitotic figures, but it is the myxoid degeneration and invasion of the muscularis propria that reliably distinguishes it from the benign counterpart.
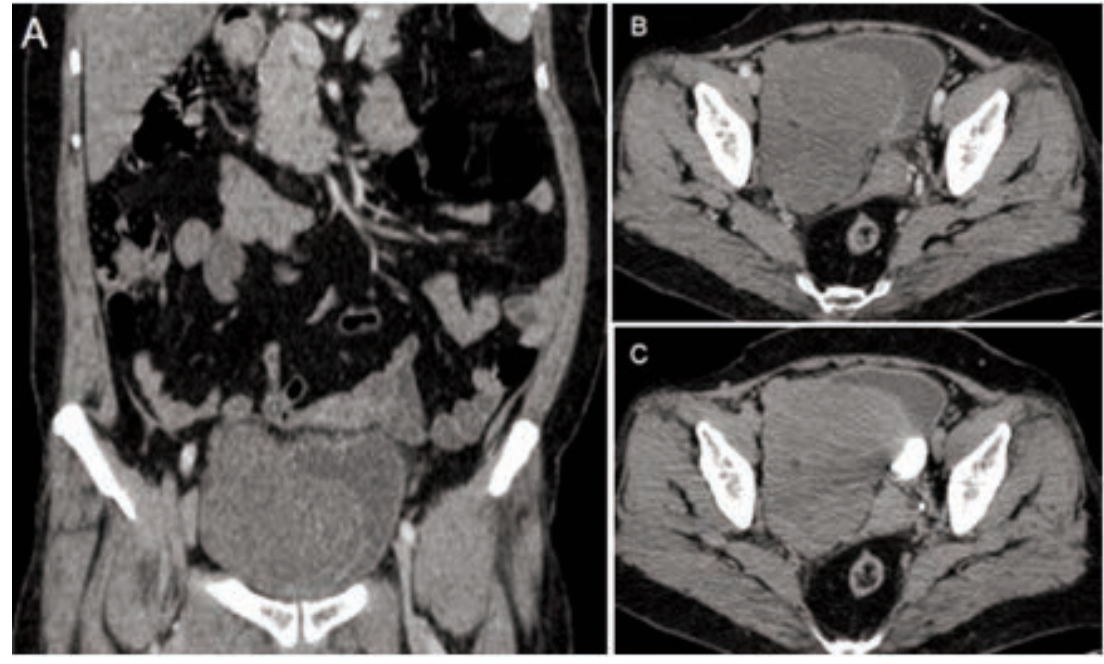

Figure 1. Coronal reformated and Axial post contrast CT images (A \& B) revealed large exophytic faintly enhanced soft tissue mass at right side of the pelvis infiltrating the right postero-lateral wall of the UB and displacing the uterus to the contralateral side, otherwise clear surrounding fat planes, it measures $13 \times 10 \times 11 \mathrm{~cm}$. Axial post contrast CT image delayed phase (C): Intact UB wall, no contrast leakage.

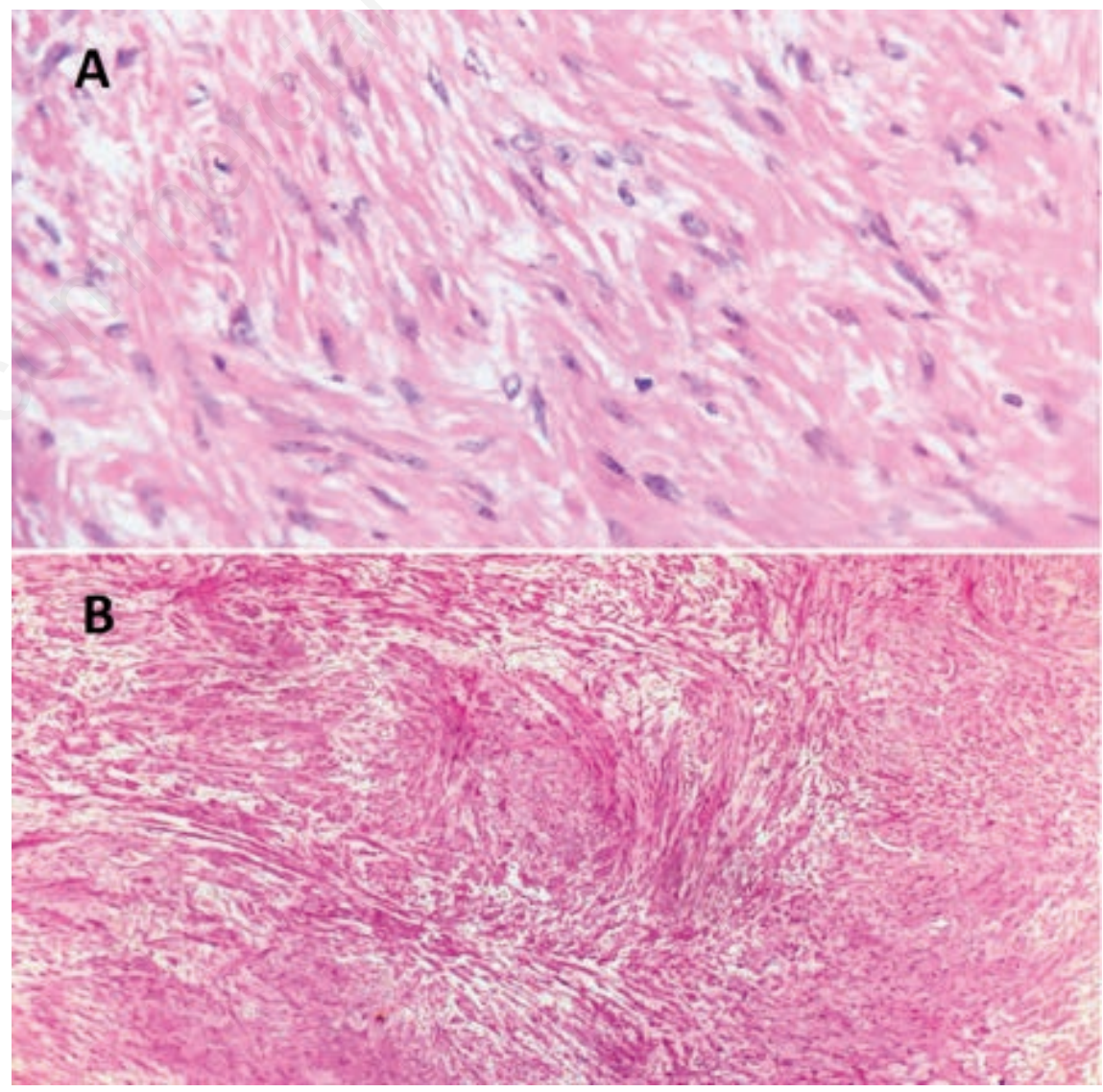

Figure 2. A) smooth muscle proliferation with cigar shaped nuclei arranged as fasicular whorly pattern $(* 400$ hematoxylin and eosin); B) leiomyoma of the bladder showing interlacing fascicles of bland spindle cells arranged in whorly pattern ( $* 4$ hematoxylin and eosin). 
Immunohistochemistry analysis of benign leiomyoma shows a positive expression for smooth muscle actin and a low expression for $\mathrm{Ki}-67 .{ }^{13}$

The prognosis of bladder leiomyoma is excellent, there are no reported cases of malignant transformation so far. ${ }^{16}$

\section{Conclusions}

Bladder leiomyoma is a rare type of bladder tumours. It usually presents with a small size of less than $10 \mathrm{~cm}$. We presented a rare case with large leiomyoma measuring $13 \mathrm{~cm}$ in its greatest dimension. She was treated by partial cystectomy with an excellent postoperative outcome.

\section{References}

1. Gok A. Transurethral resection of a large urinary bladder leiomyoma: A rare case report. Urol J 2017;14:4052-4.

2. Goktug GH, Ozturk U, Sener NC, et al. Transurethral resection of a bladder leiomyoma: A case report. Canadian Urolog Assoc J 2014;8:E111.

3. Almouhissen $\mathrm{T}$, Badr $\mathrm{H}$, Alessa $\mathrm{N}$,
Nassir A. Bladder leiomyoma in male patient presenting with renal oncocytoma: Are the two conditions related? Urology Ann 2016;8:397-9.

4. Matsushima M, Asakura H, Sakamoto $\mathrm{H}$, et al. Leiomyoma of the bladder presenting as acute urinary retention in a female patient: urodynamic analysis of lower urinary tract symptom; A case report. BMC Urol 2010;10:13.

5. Goluboff ET, O'Toole K, Sawczuk IS. Leiomyoma of bladder: report of case and review of literature. Urology 1994;43:238-41.

6. He L, Li S, Zheng C, Wang C. Rare symptomatic bladder leiomyoma: case report and literature review. J Internat Med Res 2018;46:1678-84.

7. Wu S. Imaging findings of atypical leiomyoma of the urinary bladder simulating bladder cancer: A case report and literature review. Med Ultrasonography 2013; 15:161.

8. Broessner $\mathrm{C}$, Klingler $\mathrm{CH}$, Bayer $\mathrm{G}$, et al. A 3,500-gram leiomyoma of the bladder: case report on a 3-year followup after surgical enucleation. Urologia Internat 1998;61:175-7.

9. Silva-Ramos M, Massó P, Versos R, et al. Leiomioma de vejiga: Análisis de agregación de 90 casos. Actas
Urológicas Españolas 2003;27:581-6.

10. Cornella JL, Larson T, Lee R, et al. Leiomyoma of the female urethra and bladder: report of twenty-three patients and review of the literature. Am J Obstet Gynecol 1997;176:1278-85.

11. Khater N, Sakr G. Bladder leiomyoma: Presentation, evaluation and treatment. Arab J Urol 2013;11:54-61.

12. Agrawal SK, Agrawal P, Paliwal S, Yadav C. Bladder neck leiomyoma presenting with acute retention of urine in an elderly female. J Mid-Life Health 2014;5:45.

13. Jain SK, Tanwar R, Mitra A. Bladder leiomyoma presenting with LUTS and coexisting bladder and uterine leiomyomata: a review of two cases. Rev Urol 2014;16:50.

14. Wong F, Wong Y, Ma K, Lui C. Urinary bladder leiomyoma. J Hong Kong Coll Radiol 2002;5:193-6.

15. Knoll LD, Segura JW, Scheithauer BW. Leiomyoma of the bladder. J Urology 1986;136:906-8.

16. Kim IY, Sadeghi F, Slawin KM. Dyspareunia: An unusual presentation of leiomyoma of the bladder. Rev Urol 2001;3:152. 